\title{
Clinical Study of Peripheral Arterial Disease in Lower Limbs
}

\author{
Dr. M M Rehman ${ }^{1}$, Dr.B.Anil Kumar', Dr. K.A.S.S.N.Kalyan³, \\ ${ }^{1}$ M. M Rehman M.S., General Surgery, Associate Professor, Dr.Pinnamaneni Siddhartha Institute Of Medical \\ Sciences \& Research Foundation, Chinaoutapalli, Krishna Dist, Andhra Pradesh, India. \\ ${ }^{2}$ Dr.B.Anil Kumar, M.S., General Surgery, Professor, Dr.Pinnamaneni Siddhartha Institute Of Medical \\ Sciences \& Research Foundation, Chinaoutapalli, Krishna Dist, Andhra Pradesh, India. \\ ${ }^{3}$ K. A.S.S.N.Kalyan, Junior Resident, General Surgery, Dr.Pinnamaneni Siddhartha Institute Of Medical \\ Sciences \& Research Foundation, Chinaoutapalli, Krishna Dist, Andhra Pradesh, India.
}

\begin{abstract}
:
Background: Peripheral arterial disease (PAD) is part of a global vascular problem of diffuse atherosclerosis. $P A D$ patients die mostly of cardiac and cerebrovascular-related events and much less frequently due to obstructive disease of the lower extremities. Aggressive risk factors modification is needed to reduce cardiac mortality in PAD patients.

Aim and objectives: To study the frequency of risk factors in peripheral vascular disease, To study the clinical presentations of peripheral vascular disease, To study the imaging modalities in assessment of peripheral vascular disease, To study the responsiveness of medical therapy for this disorder.

Results: It is a prospective clinical study of 50 cases of peripheral vascular disease, who are presenting to Department of Surgery, Dr.PSIMS \& RF over a period of 2 years from October'2013 to September' 2015, which included 50 admitted cases in different surgical units picked randomly and study was conducted. Atherosclerosis accounted for $80 \%$ of these cases and was found to be the commonest cause of lower limb ischemia in this region. TAO accounted for $20 \%$ and was the next common cause of lower limb ischemia. $82 \%$ cases were associated with diabetes mellitus.

Conclusion: Peripheral vascular disease is a common surgical problem with most of the population being males and among them mainly involving the smokers. Presentation was mainly claudication pain and minor percent presented with rest pain. Arterial Doppler is a specific investigation for peripheral vascular disease Patients if present early in the disease process medical line of management like exercise, antiplatelet medication, cholesterol lowering medication and drugs like Cilostazol, pentoxiphylline have been prescribed and followed. If limb salvage was planned minor procedures like debridement, ray amputation are sufficient. If they present late in the disease process Major procedures like below knee amputation and above knee amputation helped.
\end{abstract}

Keywords: peripheral arterial disease, TAO, arterial Doppler

\section{Introduction}

"Peripheral arterial disease (PAD) in the legs, sometimes known as peripheral vascular disease, is frequently caused by atheroma (fatty deposits) in the walls of the arteries, leading to insufficient blood flow to the muscles and other tissues."

Peripheral arterial disease (PAD) is part of a global vascular problem of diffuse atherosclerosis ${ }^{1}$. PAD patients die mostly of cardiac and cerebrovascular-related events and much less frequently due to obstructive disease of the lower extremities. Aggressive risk factors modification is needed to reduce cardiac mortality in PAD patients. These include smoking cessation, reduction of blood pressure to current guidelines, aggressive low density lipoprotein lowering, losing weight, controlling diabetes and the use of oral antiplatelet drugs such as aspirin or clopidogrel. In addition to quitting smoking and exercise, Cilostazol and Statins have been shown to reduce claudication in patients with PAD. Patients with critical rest limb ischemia or severe progressive claudication need to be treated with revascularization to minimize the chance of limb loss, reduce symptoms, and improve quality of life.

Patients with symptomatic peripheral arterial disease (PAD) affecting the lower extremities are initially evaluated with an ankle brachial index (ABI) and segmental pressure measurements. An ABI $<0.90$ diagnoses significant lower extremity PAD (>50\% stenosis) with a sensitivity of $79 \%$ and specificity of $96 \%{ }^{2}$. An ABI between $0.90-1.0$ is considered borderline. The localization of the stenosis can be inferred by an abnormal decrease $(>20 \mathrm{mmHg})$ in segmental lower extremity pressures. In the presence of calcified atherosclerosis, arteries may become stiff and non-compressible, which results in a falsely elevated ABI (often > 1.3). For patients with suspected PAD and a normal ABI at rest, it is valuable to obtain post-exercise $\mathrm{ABI}$ measurements which if less than 0.85 are consistent with PAD and is an independent predictor of mortality ${ }^{3}$. 
Imaging is then needed to confirm the location and degree of stenosis prior to revascularization or if the diagnosis of PAD is uncertain.

Characterization of PAD can be performed with noninvasive angiography using computed tomography (CTA) or magnetic resonance imaging (MRA) as well as with duplex ultrasonography depending on patient specific characteristics. Advances in both CTA and MRA provide clinicians with the opportunity to obtain a high resolution, 3-dimensional (3-D) road map of the peripheral arterial tree in patients particularly when planning revascularization strategies. Invasive, digital subtraction angiography (DSA) has been the gold standard for evaluation of lower extremity atherosclerosis. Although DSA is a robust technique for diagnosing significant arterial stenosis or obstruction, it provides a 2D view of the vessels, which may underestimate the degree of stenosis for tortuous vessels ${ }^{4}$. Furthermore, there are inherent risks with arterial access, ionizing radiation and the use of iodinated contrast media ${ }^{5}$.

\section{Aim and objectives:}

- To study the frequency of risk factors in peripheral vascular disease

- To study the clinical presentations of peripheral vascular disease

- To study the imaging modalities in assessment of peripheral vascular disease

- To study the responsiveness of medical therapy for this disorder .

\section{Materials and methods}

It is a prospective clinical study of 50 cases of peripheral vascular disease, who are presenting to Department of Surgery, Dr.PSIMS \& RF over a period of 2 years from October'2013 to September' 2015, which included 50 admitted cases in different surgical units picked randomly and study was conducted

\subsection{Inclusion Criteria:}

- All patients with clinical history, examination and investigations suggestive of peripheral arterial vascular disease

\subsection{Exclusion Criteria:}

- All patients with lymphatic system enlargement

- All patients who do not come for follow up

- Design : Prospective Clinical Study.

- Number of cases : 50

- Facilities : Patients were subjected to following investigations

X-rays

Ultrasound

Doppler scan

\subsection{Methods Of Collection Of Data}

1) Detailed history of the case.

2) Clinical examination.

3) Routine laboratory investigations.

4) Relevant special investigation.

5) Detailed pre-operative evaluation of the patient and appropriate preparation for surgery.

6) Surgical treatment according to the merit of the case decided by the attending surgeon under suitable anesthesia.

7) Operative findings.

8) Post-operative course, complications and their management.

9) Follow-up.

After the clinical examination, routine surgical profile along with Arterial Doppler and Lipid Profile and if necessary CT Angiography. Accordingly plan of management was decided.

At Discharge time, patient was counseled for smoking cessation, control of Diabetes and control of lipid levels, hypertension and life style modification. And advised follow-up in surgical OPD.

\section{Results}

The youngest patient was of 34 years of age, while the oldest patient was of 87 years of age. Maximum numbers of cases were observed in the age group of 51-60 years. Majority of the affected patients are male 33 (66\%). Out of 50 patients, smokers were 24(48\%), 19(38\%) were alcoholics. 16 patients were having both smoking and alcohol. Out of 50 patients, Hypertensives were 25(50\%), 41(82\%) were diabetes. 22 patients 
were having both hypertension and diabetes. Out of 50 patients, $14(28 \%)$ patients having simultaneous coronary artery disease. Almost all of them were diabetics and $60 \%$ were smokers. Out of 50 patients 27 patients presented with dry gangrene, 7 patients with ulcer, and 16 patients presented with both gangrene and ulcer. Out of 50 patients, 13 (26\%)pateints were having rest pain. And $100 \%$ of them started with claudication pain and progress to rest pain. Out of 50 patients main derangement of abnormality seen with high density lipo protein. 32 patients were having abnormal HDL. 8 patients were having high total cholesterol. And 3 patients with hightriglycerides. Out of 50 patients, $40(80 \%)$ having atherosclerosis in their doppler study. $8(16 \%)$ patients having thrombus formation and $2(4 \%)$ patients having both. Out of 50 patients, $6(12 \%)$ patients have undergone of medical line of management. And $16(32 \%)$ patients under gone minor surgery. 28(56\%) under gone major surgery.

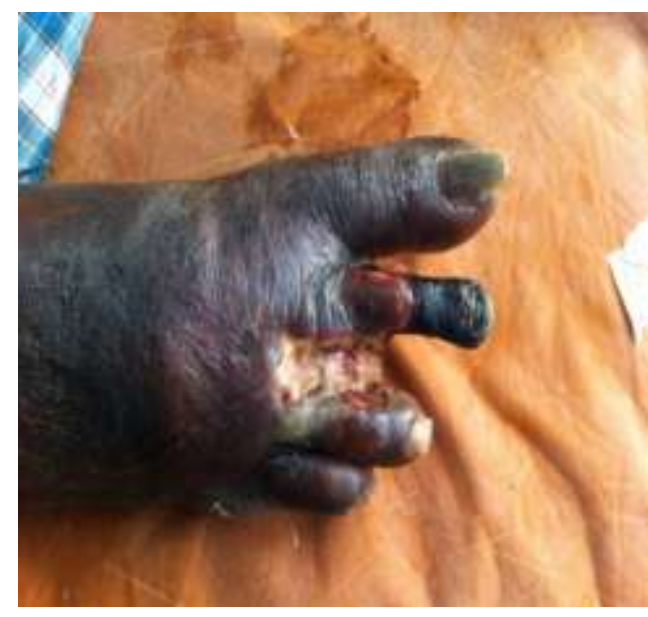

Gangrene foot with ulcer

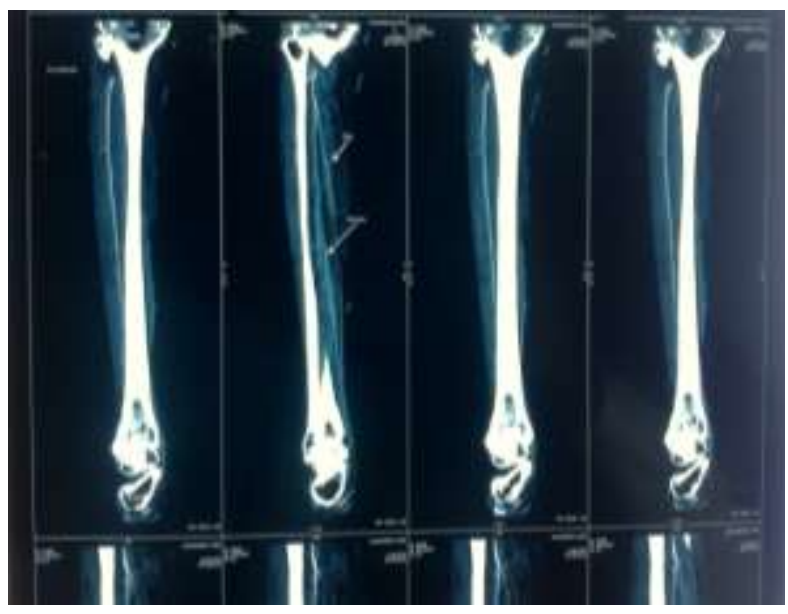

CT Angiography Picture Showing Narrowing Of Posterior Tibial Artery And Collateral Formation

Incidence:

\section{Discussion}

Fifty cases of lower limb ischemia were studied during the period of 2 years of study from 2013 to 2015 in Dr.PSIMS \& RF, Chinaoutapalli. Atherosclerosis accounted for $80 \%$ of these cases and was found to be the commonest cause of lower limb ischemia in this region. TAO accounted for $20 \%$ and was the next common cause of lower limb ischemia. $82 \%$ cases were associated with diabetes mellitus.

\section{Age Incidence:}

The youngest patient was of 34 years of age, while the oldest patient was of 87 years of age in both analyzed and studied cases. Maximum numbers of cases were observed in the age group of 51-60 years.

Sex Incidence: Among our study most of the patients were males 33(66\%), and females were $17(34 \%)$. Sex ratio is approximately 1:2 with males twice the number of females.Our study has confirmed to the accepted fact that TAO occurs exclusively in males. However in R.Nigam ET al ${ }^{6}$ (1980) study all were males except one solitary female.83\% ASO patients were males and $17 \%$ were females in both analyzed and studied cases of our study. R.Nigam et al (1980) study also reported $84 \%$ ASO patients were males and $16 \%$ were females.

Smoking \& Alcohol: Out of 50 patients, smokers were 24(48\%), 19(38\%) were alcoholics. 16 patients $(32 \%)$ were having both smoking and alcohol. Out of them 15 smokers $(30 \%)$ has about $>20$ years of exposure. 8 alcoholics $(16 \%)$ has about $>20$ years exposure.

Hypertension \& Diabetes:Out of 50 patients, Hypertensives were 25(50\%), 41(82\%) were diabetes. 22 patients were having both hypertension and diabetes.Both were independent risk factors in the development of lower limb ischemia,with diabetes being the important among. Maximum number of patients were having 
minimum 5 year duration of diabetes. Among hypertensives minimum of 6 to 10 year duration accounted for maximum patients

Coronary artery disease : Out of 50 patients, $14(28 \%)$ patients having simultaneous coronary artery disease. Almost all of them were diabetics and $60 \%$ were smokers. All the 14 patients had an attack or ischemic heart disease and were on medication.

Clinical features: In a majority of cases the onset was insidious in both TAO and ASO. But in some it followed some sort of trauma, whether it was really so or only directs the attention of the patient to the already existing disease was not certain. It was likely that the later was true.

Intermittent claudication was the presenting symptom in all the cases of TAO ( 8 out of 8 cases analyzed and cases studied) in our study. Intermittent claudication was the presenting complaint in 73 of the 200 cases of K.Inada ET al ${ }^{7}$ (1964) and 6 of the 22 cases of Szilagyi (1964). Maximum number of cases were observed (18) in the duration of 1-2 months. And least was above 6 months duration in our study.

In 30 of 40 studied cases and 23 of 50 analyzed cases intermittent claudication associated with chronic ulcer. Intermittent claudication was associated with chronic ulcer in 47 of 200 cases of K.Inada et al ${ }^{7}(1964)$. Out of 50 patients, 27 patients presented with dry gangrene, 7 patients with ulcer and 16 patients presented with both gangrene and ulcer in our study.

Gangrene with rest pain present in 13 out of 50 analyzed and 2 out of 9 studied cases of TAO in our present study. Orbon's 10 of 13 cases and K.Inada's ET al 38 of 200 cases were reported to have had ulceration and gangrene. In all these cases it was associated with rest pain. Out of 50 patients we have studied,13 (26\%)pateints were having rest pain. And $100 \%$ of them started with claudication pain and progressed to rest pain.

Involvement of the arteries: Infrapopliteal pulsations were the commonest to be absent in TAO (8 of 8 analyzed and 5 of 9 studied cases). Out of $40(80 \%)$ of atherosclerotic patients 15 patients were found to involve infra popliteal artery. And 11 patients were found to involve dorsalis pedis only.next to be involved is posterior tibial artery 7 out of 40 .

In R.Nigam et al (1980) study also the infrapopliteal pulsations were the commonest to be absent in TAO. Although, there were $11 \%$ cases of TAO with reduced or absent in TAO although, there were $11 \%$ cases of TAO with reduced or absent iliac or femoral pulses. Large vessel involvement in TAO has been reported by other workers in the Eastern and South Eastern parts of the world. ${ }^{8}$

In ASO also infra popliteal pulsations are the commonest to be absent (48 of 72 analyzed and 5 of 11 studied cases) in our present study. Absent or reduced femoral pulsations present in 12 of 72 analyzed and 5 of 11 studied cases. Arteriosclerosis of the Aorto-iliac system is infrequent in India has also shown by two studies, one from South India (Gore et al ,1960) and one from North India( Mathur et al 1961).

Investigations: A routine examination for $\mathrm{Hb} \%$, red cells, white cells, sedimentation rate, bleeding time, clotting time was done in every case. These results showing nothing special to be commented upon, except they are within normal limits.

Platelet count: Platelets were involved in thrombus formation mainly in TAO patients and to some in ASO patients. Out of 50 cases, 33 patients were having platelet count within normal limits. Only 8 (16\%)pateints were having high platelet count.

Lipid profile: Out of 50 pateints main derangement of abnormality seen with high density lipo protein. 32 (64\%)pateints were having abnormal HDL. 8 (16\%)pateints were having high total cholesterol. And 3(6\%) pateints with hightriglycerides.

In the present study consisting of TAO and ASO cases the serum cholesterol was found out to be within normal limits except that in ASO cases the mean value of serum cholesterol showed high normal.

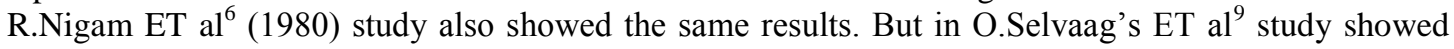
$80-90 \mathrm{mg} \%$ higher mean serum cholesterol level than our present study.

Main discrepancy we observed in our study group is low high density lipoprotein (HDL) which is altered in $32(64 \%)$ patients, other levels were of little significance.

Doppler Study: Out of 50 pateitns, $40(80 \%)$ having atherosclerosis in their doppler study. $8(16 \%)$ patients having thrombus formation and $2(4 \%)$ patints having both.Duplex scan including both flow study and velocity is the main line of investigation in conformation or diagnosing patients with lower limb ischemia. 
CT Angiography: Angiography was carried out 3 cases out of 50 patients and showed the characteristic pattern of TAO and ASO and were grouped accordingly. In our setup it was used when planning for any revascularization procedures or limb salvage surgery.

Fundoscopy: was carried out in all cases. In TAO cases fundoscopy revealed no abnormality. But in $50 \%$ of ASO cases fundoscopy revealed arterio-sclerotic changes. In R.Nigam ET al ${ }^{6}$ (1980) study fundus changes present in $30 \%$ of cases of ASO.

There are several important considerations when choosing a noninvasive imaging modality to characterize lower extremity peripheral arterial disease: 1) imaging time, 2) need for evaluation of nearby anatomy such as renovascular disease, 3 ) whether the indication is for native vessel disease or follow-up after a revascularization (bypass or stenting) and 4) patient specific factors such as kidney disease, diabetes or the presence of implantable metal devices. There are three sets of clinical guidelines which apply to the use of noninvasive imaging with lower extremity peripheral arterial disease: the European Society of Cardiology (ESC), American Heart Association (AHA)/American College of Cardiology (ACC) and TASC II.

Rapid image acquisition is strength of CTA. CTA studies can be performed with scan times $<5$ minutes compared with MRA (approximately 20-30 minutes) or Duplex ultrasonography (30-45 minutes). If both legs are evaluated by Duplex ultrasonography, the scan times are quite long (approximately 1 hour) and may hinder patient flow in the vascular lab.

Native vessel disease: The TASC II guidelines ${ }^{10}$ and $\mathrm{ESC}^{11}$ recommend obtaining an MRA, CTA or Duplex ultrasonography for imaging of native vessel disease depending upon local availability, cost and experience. American Heart Association (AHA)/American College of Cardiology (ACC) ${ }^{12}$ guidelines support the use of CTA and MRA in the diagnosis of the anatomic location and presence of significant stenosis in patients with native vessel lower extremity PAD. Imaging with either CTA or MRA is well suited for the diagnosis of patients with lower extremity PAD who have suspected aortic aneurysm/dissection, renovascular disease, trauma, or acute limb ischemia.

When choosing an imaging modality for native vessel disease, clinicians should consider if the patient has underlying renal insufficiency, diabetes or implanted metal devices. For patients who have normal renal function and are not diabetic, initial evaluation with either CTA or MRA is reasonable based on their similar excellent diagnostic capabilities. Overall, Duplex US is less sensitive technique for imaging native vessel stenosis than CTA or MRA, however the greatest limitation of Duplex US is the time required for evaluation of two lower extremities. A brief review of the important recent trials supporting the recommendation for either CTA or MRA in the diagnosis of native vessel disease follows.

A recent systematic review of the diagnostic performance of CTA included 20 studies using DSA as the gold standard ${ }^{13}$. This analysis included studies which used 2-4 detector row CT scanners as well as the contemporary 16-64 detector rows. In this population of nearly 1,000 patients, $68 \%$ had symptomatic PAD and sensitivity of CTA for stenosis $>50 \%$ or occlusion was $95 \%$ (95\% CI, $92-97 \%$ ) with a specificity of $96 \%$ (95\% CI, 93-97\%). Regardless of the location, excellent test characteristics were seen in the aorto-iliac (sensitivity $96 \%$ and specificity $98 \%$ ), femoropopliteal (sensitivity $97 \%$ and specificity $94 \%$ ) and tibial arteries (sensitivity 95\% and specificity 91\%). Traditionally, the diagnostic performance of CTA in tibial disease is lower compared to the aorta-iliac and femoral levels, particularly in the setting of heavily calcified vessels.

A more recent single-center study evaluated the diagnostic performance of 64-slice CTA compared to DSA $^{14}$ for detection of stenosis $>70 \%$ on a per segment basis. CTA had an accuracy of $98 \%$, sensitivity of $99 \%$ and specificity of $97 \%$. Furthermore, this study compared the clinical decision making outcomes using the TASC $\mathrm{II}^{15}$ guidelines based on CTA and DSA findings, which were identical in all patients but one who developed critical limb ischemia following CTA.

A recent meta-analysis was performed of contrast-enhanced MRA for determination of $>50 \%$ stenosis or occlusion in patients with symptomatic PAD ${ }^{16}$ compared to DSA. The pooled sensitivity of MRA was excellent at $95 \%$ and specificity $96 \%$. Another meta-analysis ${ }^{17}$, of CE-MRA for the diagnosis of PAD compared to DSA demonstrated multiple studies with sensitivities and specificities >95\%. Revascularization plans based on MRA agreed with the independent DSA in $90 \%$ of cases $^{18}$. Furthermore, a strategy of preoperative planning with MRA instead of DSA was expected to result in a $31 \%$ cost savings, highlighting the important role of preoperative noninvasive imaging as a cost savings measure.

Duplex ultrasonography (US) for the detection of stenosis $>50 \%$ or occlusion was evaluated in a metaanalysis of 16 studies $^{19}$ which found good sensitivity for aorto-iliac lesions (86\%) and femoropopliteal (80\%) with an excellent specificity of $96 \%$ or greater for both. In a single center study, 152 patients with symptomatic PAD were evaluated with duplex US, MRA and DSA $^{20}$. The diagnostic accuracy for haemodynamically significant stenosis involving the aorta, iliac and superficial femoral arteries was $89 \%$ for duplex US and $94 \%$ 
for MRA compared to DSA. Based on a meta-analysis, the pooled sensitivity of $88 \%$ for duplex US was significantly lower than MRA $(99 \%)$ for the detection of $\mathrm{PAD}^{21}$. However, their pooled specificities were similar (96\% for MRA and $95 \%$ for duplex US). No significant difference was noted in the diagnosis of stenosis above or below the knee ${ }^{19}$.

Given that clinicians have an option of MRA, CTA, or Duplex US for the noninvasive imaging of lower extremity PAD, it is important to evaluate the results from clinical trials comparing these modalities with regards to clinical outcomes. The Diagnostic Imaging of Peripheral Arterial Disease (DIPAD) trial evaluated clinical confidence in treatment plans and cost in over 500 patients imaged with either a Duplex US, MRA at $1.5 \mathrm{~T}$ or 16 -detector row $\mathrm{CTA}^{22}$. After adjusting for baseline variables, clinical confidence was higher and less additional imaging was required with the initial use of either MRA or CTA compared with duplex US. Importantly, the total costs for CTA were significantly lower compared to MRA or duplex US (due to the need for additional imaging in the US group). A randomized controlled trial compared CE-MRA and CTA for the initial diagnostic imaging as well as total diagnostic costs ${ }^{23}$. No difference was found in the number of patients who underwent additional vascular imaging within 60 days of their initial study. The total diagnostic cost per patient was higher for MRA due to the baseline higher cost.

Prior bypass graft: Duplex US, CTA or MRA can be used to evaluate for graft patency after lower extremity bypass surgery. The AHA/ACC recommends a surveillance program for post-op infrainguinal vein bypass grafts with duplex US at 3, 6, 12 and 24 months after surgery (Class I, LOE A). Duplex US is ideally suited for post-lower extremity arterial bypass surgery surveillance of femoral-popliteal or femoral-tibial/pedal venous grafts. Asymptomatic vein graft stenosis can result in acute thrombosis and ultimately graft failure if not detected early. In one $\operatorname{study}^{24}$, vein grafts which were revised based on stenosis findings with duplex US, had a 1 year patency rate of $90 \%$ compared to those grafts which were not revised which had a patency rate of only $66 \%$. However, Duplex US is not well established for evaluating long-term patency of angioplasty or femoralpopliteal bypass with a synthetic conduit (Class IIb, LOE B).

When Duplex US was compared with 4-row CTA, there was excellent agreement for the diagnosis of graft stenosis, aneurysmal changes and arteriovenous fistulas ${ }^{25}$. There was also no difference between Duplex US and CTA when compared with DSA. Compared to Duplex US, CTA may be superior for the detection of clinically significant stenosis in peripheral arterial disease bypass grafts ${ }^{25}$

Stents: CTA is superior to MRA for evaluation of restenosis in metallic stents or stent grafts. There are varying types of metal vascular stents, which influence the ability of a MRA to reliably determine the degree of in-stent restenosis ${ }^{26}$. The lumen within a steel stent may be completely obscured due to susceptibility artifact and radiofrequency shielding. Stents made with nitinol, cobalt and platinum are less sensitive to these effects. Stent oriented with long axis parallel to the B0 field of the scanner demonstrate better imaging characteristic as compare to those oriented perpendicular.

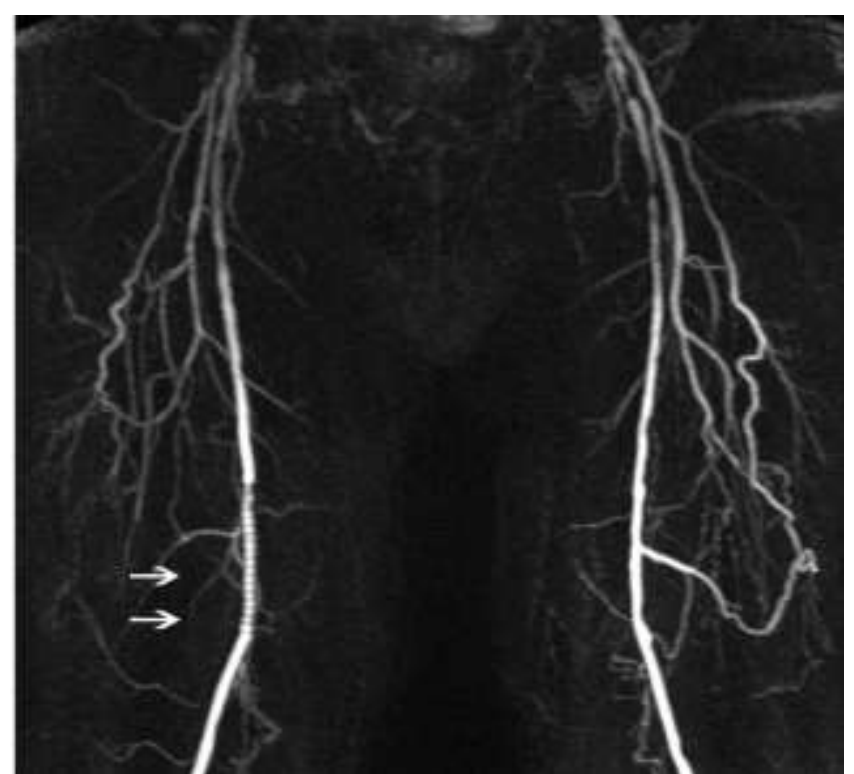

Fig: MRA showing a clearly patent right superficial femoral artery stent (double arrows). This is a nitinol stent which doesn't have the metal dropout artifact on MRI. 
$\mathrm{Li}$ et al. used 64-detector row $\mathrm{CTA}^{27}$ to determine in-stent restenosis in 41 patients compared with DSA. Of the 81 stents, $76 \%$ were considered to be assessable based on image quality; motion or metal artifact influenced the interpretability of the other stents. For the group of evaluable stents, there was excellent sensitivity (95\%) and specificity (96\%) for detection of in-stent stenosis. When comparing the total group of in-stent stenosis seen on DSA with all CTA images (evaluable and non-evaluable stents), there was a lower sensitivity of $86 \%$ and specificity of $72 \%$ for CTA detection of in-stent stenosis. Beam hardening artifact from stents still limits detection of in-stent restenosis with CTA. Future in vivo studies will be required to determine if iterative reconstruction can improve visualization.

Duplex US can be used to determine areas of in-stent restenosis, particularly within the iliac, superficial femoral artery or popliteal arteries. To identify $>80 \%$ stenosis of the superficial femoral artery compared to $\mathrm{DSA}^{28}$, a peak systolic velocity $>275 \mathrm{~cm} / \mathrm{s}$ and velocity ratio (comparing the velocity within the stent to a diseased free area vessel proximal to the stent) of $>3.5$ has excellent specificity (94\%) but only moderate sensitivity $(74 \%)$. The limited sensitivity of Duplex US for detection of significant stenosis results in CTA being the diagnostic modality of choice for in-stent restenosis unless there are concerns regarding the potential for renal failure with the iodinated contrast media.

\section{Special Populations}

Diabetic Patients : Patients with diabetes often have heavily calcified vessels, which presents a diagnostic challenge in terms of using duplex US or CTA. With duplex US, heavily calcified vessels may result in an obscured arterial lumen and inability to reliably diagnose the degree of PAD. CE-MRA is not subject to image artifact generated by heavily calcified vessels. Inter-observer agreement for degree of stenosis or occlusion is higher with CE-MRA than CTA, in the presence of arterial calcifications ${ }^{29}$. From our perspective, for diabetic patients, MRA should be considered the test of choice due to calcified vessels and concomitant renal insufficiency.

Renal Failure :As discussed previously, due to concerns regarding NSF, the current recommendation is to avoid GBCA's in patients with advanced renal failure with an estimated glomerular filtration rate (GFR) < $30 \mathrm{ml} / \mathrm{min} / 1.73 \mathrm{~m}^{2}$ unless the information provided by the contrast-enhanced MRI is not obtainable by another means. Patients with any degree of renal insufficiency can be safely imaged with Duplex US and non-contrast MRA. With CTA, iodinated contrast media has an associated risk of contrast-induced nephropathy (CIN) defined as an increase in serum creatinine from baseline by $>25 \%$ or $>0.5 \mathrm{mg} / \mathrm{dl}$ within 3 days of contrast agent administration without other cause. Those at highest risk for CIN have baseline renal insufficiency, diabetes, concurrent nephrotoxic drug use, hypertension, congestive heart failure and dehydration. Large doses of contrast media and high-osmolar contrast media may further increase the risk of CIN. The greatest risk seems to be in patients with preexisting renal insufficiency, particularly those with diabetes, regardless of the osmolality of the contrast agent used it is important to ensure adequate hydration and to minimize the total volume of contrast media used. In addition, patients with allergic reactions to iodinated contrast media need pre-medication with steroids and antihistamine prior to receiving it.

Implanted Metal Devices : For patients with implanted metal devices, it is important to determine compatibility with exposure to magnetic fields. Device information cards may have manufacturer information regarding safety testing with MRI. Publications are available which consolidate the available information regarding metal devices and MRI safety. Newer pacemakers are being developed that are MRI-compatible. Patients who are pacemaker-dependent or have certain types of brain aneurysm clips, neurostimulator devices or retained ocular ferromagnetic foreign bodies are best imaged with another modality due to potential risk. In addition, even if an implanted medical device is considered compatible with the MRI environment there may be significant susceptibility artifact generated during the scan.

Clinical Application Summary:Given the options available for multi-modality imaging of lower extremity PAD, we present an overview based on our perspective and review of the literature. 


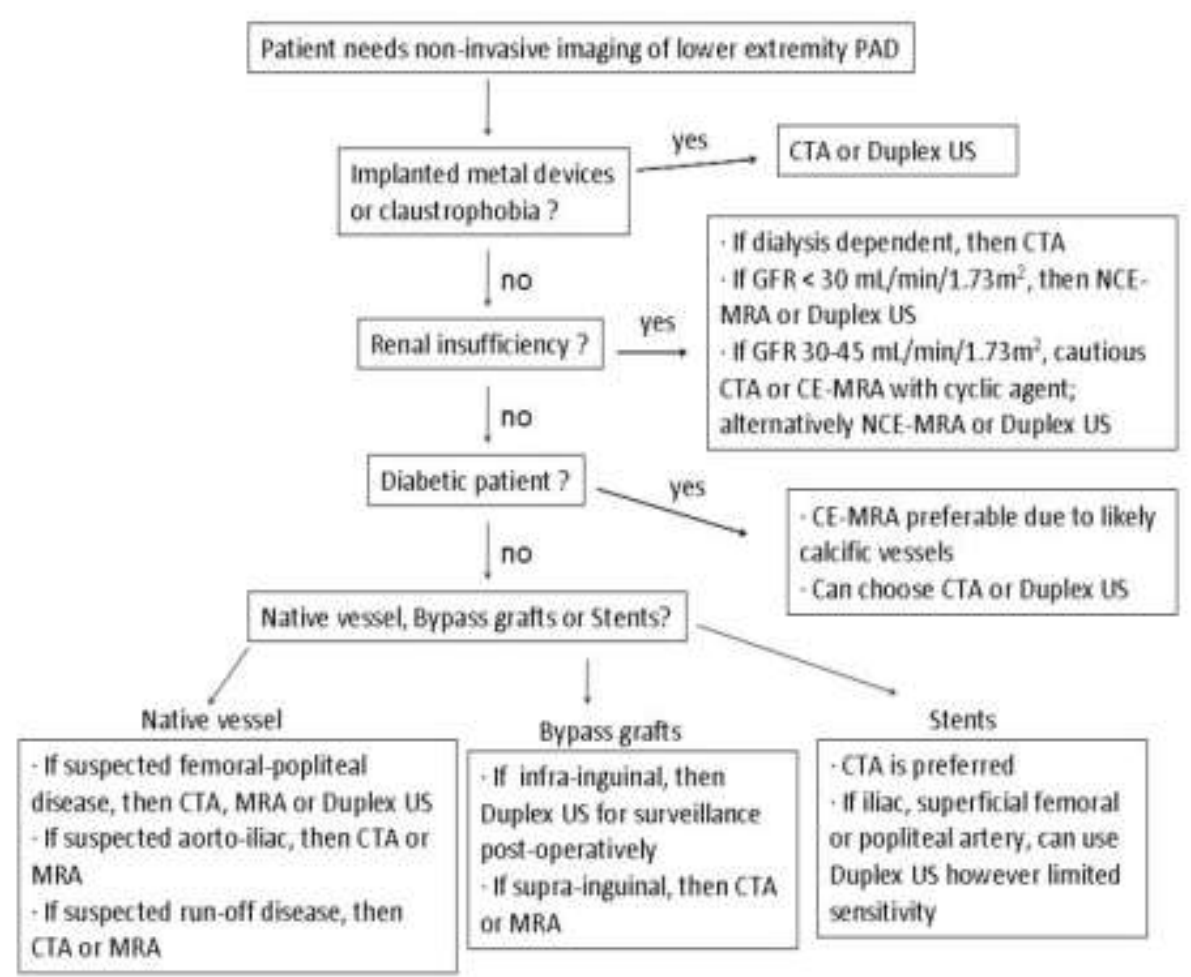

Figure showing an algorithm for choosing the appropriate imaging modality for a given clinical scenario.

\section{Treatment:}

Medical: Medical line of treatment was instituted in all patients soon after admission. Out of 50 patients, $6(12 \%)$ patients have undergone of medical line of management. Analgesics, Sedatives, Haematinics were given whenever necessary. Specific drugs like Cilostazol, which acts on blood vessels and pentoxiphylline, which acts by modifying the shape of the red blood corpuscles were used. Conservative management includes observation mainly, and daily dressings of the wound whether it is dry gangrene or ulcer or both.

After reviewing 100 cases treated by placental implantation, E.Troensegaard Hansen has reported very encouraging results, even in cases when lumbar sympathectomy was not done. Similar reports were made by Gow (1956), Rowling (1958) etc. But Moore (1957) has found discouraging results. Kinmonth et al (1962) suggests that this line of treatment must be subjected to the strictest scrutiny before the claim can be accepted.

\section{Surgical:}

Amputation of Digits: 28(56\%) patients under gone minor surgery like Ray amputation, or disarticulation of the involved toe .This modality was made use for patients with early stages of lower limb ischemia . Some patients disease did not progress and remained stable and remaining it progressed and needed major surgery like amputation of the involved limb.

Amputation of Limb: 16 (32\%) patients under gone major surgery.13 out of 16 have undergone below knee Amputations 11 in ASO and 2 in TAO. Above knee Amputations were carried out in 3 cases, all were ASO. There was relief from pain and other symptoms in all cases.

\section{Conclusion}

Peripheral vascular disease is a common surgical problem with most of the population being males and among them mainly involving the smokers. Pertaining to smokers, consuming beedi was found to increase the risk of vascular disease profoundly in contrary to cigarettes. There is an increasing trend towards the diabetic population and there is a steady trend among smokers. Any previous history of coronary artery disease should align towards diffuse vascular disease mainly atherosclerosis.

Presentation was mainly claudication pain and minor percent presented with rest pain. These both give an idea regarding the severity of vessel involvement and also regarding the management. Peripheral pulse examination plays a pivotal role in peripheral vascular disease, here mainly the infrapopletial group of vessels was involved most compare to others inferring peripheral vascular disease starts involving the distal vessels first. 
Blood investigations routinely does not contribute to the management protocol, but specifically platelet count, blood sugar, lipid profile should be accounted for. High platelet count high blood sugars and altered high density lipoprotein were important among them.

Arterial Doppler is a specific investigation for peripheral vascular disease and is a must for every patient with features of arterial disease. Doppler study helps in evaluating velocity and phasic flow of the vessel. If in doubt, or is planning for any revascularization procedures CT Angio has been planned.

Patients if present early in the disease process medical line of management like exercise, antiplatelet medication, cholesterol lowering medication and drugs like Cilostazol, pentoxiphylline have been prescribed and followed. If limb salvage was planned minor procedures like debridement, ray amputation are sufficient. If they present late in the disease process Major procedures like below knee amputation and above knee amputation helped. So, the line of management and selection of patient is critical for high efficient outcome.

\section{References}

[1]. Shammas NW. Epidemiology, classification, and modifiable risk factors of peripheral arterial disease. Vascular Health and Risk Management. 2007; 3(2):229-234.

[2]. Lijmer JG, Hunink MG, van den Dungen JJ, Loonstra J, Smit AJ. ROC analysis of noninvasive tests for peripheral arterial disease. Ultrasound Med Biol. 1996; 22:391-398.

[3]. Sheikh MA, Bhatt DL, Li J, Lin S, Bartholomew JR. Usefulness of Post Exercise Ankle-Brachial Index to Predict All-Cause Mortality. Am J Cardiol. 2011; 107:778-782.

[4]. Ota H, Takase K, Rikimaru H, Tsuboi M, Yamada T, Sato A, Higano S, Ishibashi T, Takahashi S. Quantitative Vascular Measurements in Arterial Occlusive Disease1. Radio graphics. 2005; 25:1141-1158.

[5]. Norgren L, Hiatt WR, Dormandy JA, Nehler MR, Harris KA, Fowkes FG. On behalf of the TASC II Working group. Inter-society consensus for the management of peripheral arterial disease (TASC II) J Vasc Surg. 2007; 45:S5-S67.

[6]. R.Nigam \& Kosovichev A.G. Ind.J.Surg 1980;:9-15.

[7]. Inada K, Hirose M, Iwashima Y, Matsumoto K. Popliteal artery entrapment syndrome: a case report. Br J Surg 1978; 65:613-5.

[8]. GoodmanSJ et al. The Diabetic Neuropathies .Use of Lateral and medial flap. Arch.Surg.1965; 91:506. Selvag O, etal. Progressive tendency of arteriosclerosis obliterans of the lower extremities Acta Chir Scand (Suppl) 1960; 253:187-95.

[9]. Norgren L, Hiatt WR, Dormandy JA, Nehler MR, Harris KA, Fowkes FG. On behalf of the TASC II Working group. Inter-society consensus for the management of peripheral arterial disease (TASC II) J Vasc Surg. 2007; 45:S5-S67.

[10]. Tendera M, Aboyans V, Bartelink ML, Baumgartner I, Clement D, Collet JP, Cremonesi A, De Carlo M, Erbel R, Fowkes FG, Heras M, Kownator S, Minar E, Ostergren J, Poldermans D, Riambau V, Roffi M, Rother J, Sievert H, van Sambeek M, Zeller T, Bax J, Auricchio A, Baumgartner H, Ceconi C, Dean V, Deaton C, Fagard R, Funck-Brentano C, Hasdai D, Hoes A, Knuuti J, Kolh P, McDonagh T, Moulin C, Poldermans D, Popescu B, Reiner Z, Sechtem U, Sirnes PA, Torbicki A, Vahanian A, Windecker S, Kolh P, Torbicki A, Agewall S, Blinc A, Bulvas M, Cosentino F, De Backer T, Gottsater A, Gulba D, Guzik TJ, Jonsson Br, Kesmarky G, Kitsiou A, Kuczmik W, Larsen ML, Madaric J, Mas JL, McMurray JJV, Micari A, Mosseri M, Muller C, Naylor R, Norrving B, Oto O, Pasierski T, Plouin PF, Ribichini F, Ricco JB, Ruilope L, Schmid JP, Schwehr U, Sol BGM, Sprynger M, Tiefenbacher C, Tsioufis C, Van Damme H. ESC Guidelines on the diagnosis and treatment of peripheral artery diseases. Eur Heart J. 2011;32:2851-2906

[11]. Rooke TW, Hirsch AT, Misra S, Sidawy AN, Beckman JA, Findeiss LK, Golzarian J, Gornik HL, Halperin JL, Jaff MR, Moneta GL, Olin JW, Stanley JC, White CJ, White JV, Zierler RE. 2011 ACCF/AHA Focused Update of the Guideline for the Management of Patients with Peripheral Artery Disease (Updating the 2005 Guideline): A Report of the American College of Cardiology Foundation/American Heart Association Task Force on Practice Guidelines. J Am Coll Cardiol. 2011; 58:2020-2045.

[12]. Rooke TW, Hirsch AT, Misra S, Sidawy AN, Beckman JA, Findeiss LK, Golzarian J, Gornik HL, Halperin JL, Jaff MR, Moneta GL, Olin JW, Stanley JC, White CJ, White JV, Zierler RE. 2011 ACCF/AHA Focused Update of the Guideline for the Management of Patients with Peripheral Artery Disease (Updating the 2005 Guideline): A Report of the American College of Cardiology Foundation/American Heart Association Task Force on Practice Guidelines. J Am Coll Cardiol. 2011; 58:2020-2045.

[13]. Napoli A, Anzidei M, Zaccagna F, Cavallo Marincola B, Zini C, Brachetti G, Cartocci G, Fanelli F, Catalano C, Passariello R. Peripheral Arterial Occlusive Disease: Diagnostic Performance and Effect on Therapeutic Management of 64-Section CT Angiography. Radiology. 2011; 261:976-986.

[14]. Norgren L, Hiatt WR, Dormandy JA, Nehler MR, Harris KA, Fowkes FG. on behalf of the TASC II Working group. Inter-society consensus for the management of peripheral arterial disease (TASC II) J Vasc Surg. 2007; 45:S5-S67.

[15]. Menke J, Larsen J. Meta-analysis: Accuracy of Contrast-Enhanced Magnetic Resonance Angiography for Assessing Stenoocclusions in Peripheral Arterial Disease. Ann Int Med. 2010; 153:325-334.

[16]. Dellegrottaglie S, Sanz J, Macaluso F, Einstein AJ, Raman S, Simonetti OP, Rajagopalan S. Technology Insight: magnetic resonance angiography for the evaluation of patients with peripheral artery disease. Nat Clin Pract Cardiovasc Med. 2007; 4:677687.

[17]. Hoch JR, Tulles MJ, Kennell TW, McDermott J, Acher CW, Turnipseed WD. Use of magnetic resonance angiography for the preoperative evaluation of patients with infrainguinal arterial occlusive disease. J Vasc Surg. 1996; 23:792-801.

[18]. Koelemay MJ, den Hartog D, Prins MH, Kromhout JG, Legemate DA, Jacobs MJ. Diagnosis of arterial disease of the lower extremities with duplex ultrasonography. Br J Surg. 1996; 83:404-409.

[19]. Leiner T, Kessels AGH, Nelemans PJ, Vasbinder GB, de Haan MW, Kitslaar PEJH, Ho KY, Tordoir JHM, van Engelshoven JMA Peripheral Arterial Disease: Comparison of Color Duplex US and Contrast-enhanced MR Angiography for Diagnosis. Radiology. 2005; 235:699-708.

[20]. Visser K, Hunink MGM. Peripheral arterial disease: gadolinium-enhanced MR angiography versus color-guided duplex US - a meta-analysis. Radiology. 2000; 216:67-77.

[21]. Ouwendijk R, de Vries M, Stijnen T, Pattynama PMT, van Sambeek MRHM, Buth J, Tielbeek AV, van der Vliet DA, SchutzeKool LJ, Kitslaar PJEH, de Haan MW, van Engelshoven JMA, Hunink MGM. For the Program for the Assessment of Radiological Technology. Multicenter Randomized Controlled Trial of the Costs and Effects of Noninvasive Diagnostic Imaging in Patients with Peripheral Arterial Disease: The DIPAD Trial. AJR American Journal of Roentgenology. 2008; 190:1349-1357. 
[22]. Ouwendijk R, de Vries M, Pattynama PMT, van Sambeek MRHM, de Haan MW, Stijnen T, van Engelshoven JMA, Hunink MGM. Imaging Peripheral Arterial Disease: A Randomized Controlled Trial Comparing Contrast-enhanced MR Angiography and MultiDetector Row CT Angiography. Radiology. 2005; 236:1094-1103.

[23]. Mattos MA, van Bemmelen PS, Hodgson KJ, Ramsey DE, Barkmeier LD, Sumner DS. Does correction of stenosis identified with color duplex scanning improve infrainguinal graft patency? J Vasc Surg. 1993; 17:54-64.

[24]. Willmann JK, Mayer D, Banyai M, Desbiolles LM, Verdun FR, Seifert B, Marincek B, Weishaupt D. Evaluation of Peripheral Arterial Bypass Grafts with Multi- Detector Row CT Angiography: Comparison with Duplex US and Digital Subtraction Angiography1. Radiology. 2003; 229:465-474.

[25]. Roditi G, Kusumawidjaja D. Magnetic resonance angiography and computed tomography angiography for peripheral arterial disease. Imaging. 2009; 21:85-108.

[26]. Li Xm, Li Yh, Tian Jm, Xiao Y, Lu Jp, Jing Zp, Sheng J, Edwin A, Wu Fh. Evaluation of peripheral artery stent with 64-slice multidetector row CT angiography: Prospective comparison with digital subtraction angiography. Euro J Radiol. 2010; 75:98-103.

[27]. Baril DT, Rhee RY, Kim J, Makaroun MS, Chaer RA, Marone LK. Duplex criteria for determination of in-stent stenosis after angioplasty and stenting of the superficial femoral artery. J Vasc Surg. 2009; 49:133-139.

[28]. Ouwendijk R, Kock MCJM, Visser K, Pattynama PMT, de Haan MW, Hunink MGM. Interobserver Agreement for the Interpretation of Contrast-Enhanced 3D MR Angiography and MDCT Angiography in Peripheral Arterial Disease. AJR American Journal of Roentgenology. 2005;185:1261-1267

[29]. Kribben A, Witzke O, Hillen U, Barkhausen J, Daul AE, Erbel R. Nephrogenic systemic fibrosis: pathogenesis, diagnosis and therapy. J Am Coll Cardiol. 2009; 53:1621-1628. 